Directeur de LA PUblication / Publication diRECTOR: Bruno David,

Président du Muséum national d'Histoire naturelle

RÉDACTEUR EN CHEF / EDITOR-IN-CHIEF: Jean-Philippe Siblet

ASSISTANTE DE RÉDACTION / ASSISTANT EDITOR: Sarah Figuet (naturae@mnhn.fr)

Mise EN PAGE / PAGE LAYOUT: Sarah Figuet

COMITÉ SCIENTIFIQUE / SCIENTIFIC BOARD:

Luc Abbadie (UPMC, Paris)

Luc Barbier (Parc naturel régional des caps et marais d'Opale, Colembert)

Aurélien Besnard (CEFE, Montpellier)

Vincent Boullet (Expert indépendant flore/végétation, Frugières-le-Pin)

Hervé Brustel (École d'ingénieurs de Purpan, Toulouse)

Patrick De Wever (MNHN, Paris)

Thierry Dutoit (UMR CNRS IMBE, Avignon)

Éric Feunteun (MNHN, Dinard)

Romain Garrouste (MNHN, Paris)

Grégoire Gautier (DRAAF Occitanie, Toulouse)

Olivier Gilg (Réserves naturelles de France, Dijon)

Frédéric Gosselin (Irstea, Nogent-sur-Vernisson)

Patrick Haffner (UMS PatriNat, Paris)

Frédéric Hendoux (MNHN, Paris)

Xavier Houard (OPIE, Guyancourt)

Isabelle Leviol (MNHN, Concarneau)

Francis Meunier (Conservatoire d'espaces naturels - Hauts-de-France, Amiens)

Serge Muller (MNHN, Paris)

Francis Olivereau (DREAL Centre, Orléans)

Laurent Poncet (UMS PatriNat, Paris)

Nicolas Poulet (OFB, Vincennes)

Jean-Philippe Siblet (UMS PatriNat, Paris)

Laurent Tillon (ONF, Paris)

Julien Touroult (UMS PatriNat, Paris)

COUVERTURE / COVER:

Prélèvement de fèces de Desman des Pyrénées Galemys pyrenaicus (É. Geoffroy Saint-Hilaire, 1811). Crédit photo: Lucas Santucci - Agence Zeppelin.

Naturae est une revue en flux continu publiée par les Publications scientifiques du Muséum, Paris

Naturae is a fast track journal published by the Museum Science Press, Paris

Les Publications scientifiques du Muséum publient aussi / The Museum Science Press also publish:

Adansonia, Zoosystema, Anthropozoologica, European Journal of Taxonomy, Geodiversitas, Cryptogamie sous-sections Algologie, Bryologie, Mycologie, Comptes Rendus Palevol.

Diffusion - Publications scientifiques Muséum national d'Histoire naturelle

CP $41-57$ rue Cuvier F-75231 Paris cedex 05 (France)

Tél. : 33 (0)1407948 05 / Fax: 33 (0)1 40793840

diff.pub@mnhn.fr / https://sciencepress.mnhn.fr

(c) Publications scientifiques du Muséum national d'Histoire naturelle, Paris, 2021

ISSN (électronique / electronic): 1638-9387 


\title{
Étude comparative de la densité et du déplacement des Desmans des Pyrénées Galemys pyrenaicus (É. Geoffroy Saint-Hilaire, 1811) par une méthode non invasive
}

\author{
Melody LIM \\ Conservatoire d'Espaces naturels d'Occitanie, \\ 75 voie du TOEC, F-31076 Toulouse Cedex 3 (France) \\ melody.lim@cen-occitanie.org \\ Frédéric BLANC \\ Conservatoire d'Espaces naturels d'Occitanie, \\ 75 voie du TOEC, F-31076 Toulouse Cedex 3 (France) \\ et GEODE CNRS, Université Toulouse - Jean Jaurès, Maison de la Recherche, \\ 5 allée Antonio-Machado, F-31058 Toulouse Cedex 9 (France) \\ frederic.blanc@cen-occitanie.org
}

Johan MICHAUX

Lise-Marie PIGNEUR

François GILLET

Laboratoire de Génétique de la Conservation (GeCoLAB), Institut de Botanique (Bâtiment B22) Quartier Vallée 1, Chemin de la vallée 4, B-4000 Liège (Sart Tilman) (Belgique) contact.gecolab@gmail.com

Daniel MARC

Conservatoire d'Espaces naturels d'Occitanie, 75 voie du TOEC, F-31076 Toulouse Cedex 3 (France) et GEODE CNRS, Université Toulouse - Jean Jaurès, Maison de la Recherche, 5 allée Antonio-Machado, F-31058 Toulouse Cedex 9 (France) daniel.marc@cen-occitanie.org

Émile PONCET
Mélanie NÉMOZ
Conservatoire d'Espaces naturels d'Occitanie,
75 voie du TOEC, F-31076 Toulouse Cedex 3 (France)
emile.poncet@cen-occitanie.org
melanie.nemoz@cen-occitanie.org

Soumis le 9 mai 2020 | Accepté le 17 janvier 2021 | Publié le 4 août 2021

Lim M., Blanc F., Michaux J., Pigneur L.-M., Gillet F., Marc D., Poncet É. \& Némoz M. 2021. - Étude comparative de la densité et du déplacement des Desmans des Pyrénées Galemys pyrenaicus (É. Geoffroy Saint-Hilaire, 1811) par une méthode non invasive. Naturae 2021 (17): 233-242. https://doi.org/10.5852/naturae2021a17

RÉSUMÉ

Espèce protégée et en régression sur l'ensemble de son aire de répartition, le Desman des Pyrénées Galemys pyrenaicus (É. Geoffroy Saint-Hilaire, 1811) reste une espèce mal connue malgré les programmes d'actions en faveur de sa conservation mis en œuvre en France depuis 2010. Le manque de connaissance sur la densité des populations et les déplacements des individus nous ont poussés à réaliser une étude de suivi de six populations de Desman répartie sur six sites pyrénéens connus pour abriter l'espèce. La méthode retenue pour cette étude a été l'analyse génétique des fecces nécessitant 


\section{MOTS CLÉS \\ Galemys pyrenaicus, génétique,
fèces, utilisation de l'espace,} densité de population.

\section{KEY WORDS \\ Galemys pyrenaicus, genetic, \\ faeces, \\ space use,}

population density. un protocole non invasif et moins coûteux que les captures ou les suivis par radiopistage. Certaines crottes ont permis de contacter des individus à plusieurs reprises et d'analyser leurs déplacements. L'individualisation des crottes a également permis d'estimer une densité minimale d'individus sur $1,5 \mathrm{~km}$ de cours d'eau prospecté réparti sur $3 \mathrm{~km}$, ou encore sur des portions plus restreintes de 250 mètres. Ainsi, les résultats confirment la mobilité du Desman mais aussi son caractère nomade et non territorial. Un des sites n'a cependant pas pu rentrer dans l'analyse des données, car soumis à une pollution menant à la disparition de l'espèce pendant quelques années. Les analyses génétiques ont montré l'efficacité des prospections avec des résultats positifs au Desman pour chaque échantillon prélevé supposé appartenir à l'espèce. Cependant, ils confirment aussi la difficulté de détectabilité de l'espèce malgré sa présence et la nécessité de la prendre en compte, même si elle semble absente à un instant donné sur un site, notamment dans les projets d'aménagement de cours d'eau, afin de respecter la continuité écologique nécessaire à ses déplacements et aux mélanges génétiques des populations.

\begin{abstract}
Comparative study of the density and movement of Pyrenean desmans Galemys pyrenaicus (É. Geoffroy Saint-Hilaire, 1811) using a non-invasive method.

Protected and declining throughout its range, the Pyrenean Desman Galemys pyrenaicus (É. Geoffroy Saint-Hilaire, 1811) remains a poorly known species despite two conservation programs led in its favor in France since 2010. The lack of knowledge about the density of its populations and the individual movements encouraged us to carry out a study of six Desman populations distributed over six Pyrenean sites known to host the species. The method chosen was the genetic analysis of faeces, a non-invasive and less costly protocol than capture or radio tracking. Analyses of individual movements were possible by contacting individual faeces on several occasions. Faeces which allowed genetic analyses to identify an individual Desman made it possible to estimate a minimum density of desmans over $1.5 \mathrm{~km}$ prospected river spread over $3 \mathrm{~km}$ or even over more restricted portions of 250 meters. Thus, these results confirm the mobility of the Desman but also its nomadic and non-territorial nature. Nevertheless, one of the sites was excluded of the analyses since it was hit by a pollution leading to the disappearance of the species for a few years. Genetic analyses pointed out the efficiency of the faeces collecting protocol with positive results for each sample taken assumed to belong to the species. However, they also confirm the difficulty of the species detectability despite its presence. This means that even if the Pyrenean Desman seems to be absent of a river at a given time, we still need to take it into account. Likewise, it is necessary to take it into account in every river management projects in order to respect the ecological continuity necessary for its movements and the genetic mixing of its populations.
\end{abstract}

\section{INTRODUCTION}

Malgré les programmes d'actions en faveur du Desman des Pyrénées Galemys pyrenaicus (É. Geoffroy Saint-Hilaire, 1811) mis en œuvre depuis 2010, beaucoup d'éléments de sa biologie restent encore à élucider. Les études de radiopistage menées sur l'espèce (Stone 1985; Chora \& Quaresma 2001; Melero et al. 2011, 2014; Lim 2015; Esnaola et al. 2018) ont permis l'acquisition de connaissances telles que les linéaires de cours d'eau utilisés sur une période de suivi, les rythmes d'activité ou encore la localisation des gîtes fréquentés. Cependant, les apports en connaissances relatives à la densité des populations, leurs interactions ou leurs déplacements sur de plus grandes périodes de temps, sont peu nombreux (Nores et al. 1998; Gisbert \& García-Perea 2004; Gillet et al. 2016; Escoda et al. 2018).

Ce manque de connaissance, le statut «Vulnérable» du Desman des Pyrénées sur les Listes rouges mondiale (https://www. iucnredlist.org/species/8826/114145177, dernière consultation le 30 juillet 2020) et nationale (UICN France et al. 2017), son état de conservation "Défavorable mauvais» (https://inpn. mnhn.fr/espece/cd_nom/60243/tab/statut, dernière consultation le 30 juillet 2020) sur l'ensemble des régions où il est présent (alpine, atlantique et méditerranéenne) et sa régression sur l'ensemble de son aire de répartition (Gisbert \& GarcíaPerea 2014; Aymerich \& Gosálbez 2015, 2018; Charbonnel 2015; Poncet et al. 2017; Quaglietta et al. 2018) ont motivé le Conservatoire d'espaces naturels d'Occitanie et ses partenaires à s'interroger sur le fonctionnement de populations de Desman des Pyrénées réparties sur six cours d'eau pyrénéens entre 2014 et 2016. Pour ce faire, l'analyse génétique des crottes a été la méthode la plus pertinente du fait de son caractère non invasif et de la pression humaine moindre comparée à des opérations de capture ou de radiopistage (Poncet et al. 2021).

Les données acquises dans le cadre des programmes de conservation du Desman des Pyrénées (Némoz \& Bertrand 2009; Gillet 2015) et par les chercheurs catalans laissent supposer une espèce à l'abondance locale forte, non territoriale et nomade (Aymerich \& Gosálbez 2014; Escoda et al. 2018).

L'étude ayant été faite dans le cadre du programme LIFE+ Desman, les sites devaient se situer en zone Natura 2000. Il a été possible de choisir six sites de contextes variables. 
TABLEAU 1. - Présentation des caractéristiques de chaque site d'étude.

\begin{tabular}{|c|c|c|c|}
\hline $\begin{array}{l}\text { Cours d'eau } \\
\text { et site Natura2000 }\end{array}$ & Localisation & Altitude & Caractéristiques du cours d'eau \\
\hline $\begin{array}{l}\text { Salat } \\
\text { FR7301822 }\end{array}$ & $\begin{array}{l}\text { Commune d'Ustou à proximité } \\
\text { du lieu-dit «Pont de la Taule» }\end{array}$ & $\begin{array}{l}600 \mathrm{~m} \\
\quad(100 \mathrm{~m} \text { de dénivelé) }\end{array}$ & $\begin{array}{l}\text { Hétérogénéité des faciès d'écoulement: } \\
\text { - courant rapides et petites cascades avec } \\
\text { gros blocs émergents; } \\
\text { - radiers avec petits blocs, galets et graviers; } \\
\text { - plats courants aux substrats plus sableux. }\end{array}$ \\
\hline $\begin{array}{l}\text { Rébenty } \\
\text { FR9101468 }\end{array}$ & Belfort-sur-Rébenty & $\begin{array}{l}700 \mathrm{~m} \\
\quad(30 \mathrm{~m} \text { de dénivelé) }\end{array}$ & $\begin{array}{l}\text { Faciès d'écoulement plutôt uniforme. } \\
\text { Aucun obstacle, aucun seuil ni aucun } \\
\text { aménagement. }\end{array}$ \\
\hline $\begin{array}{l}\text { Nohèdes } \\
\text { FR9101473 }\end{array}$ & $\begin{array}{l}\text { Rivière Nohèdes } \\
\text { et ruisseau de Torreilles }\end{array}$ & $\begin{array}{l}1000 \mathrm{~m} \\
\quad(320 \mathrm{~m} \text { de dénivelé) }\end{array}$ & $\begin{array}{l}\text { En amont et en aval de la centrale } \\
\text { hydroélectrique. Sous le point de restitution } \\
\text { de l'eau à la centrale (deux sections) et en } \\
\text { amont de la centrale. }\end{array}$ \\
\hline $\begin{array}{l}\text { Aston } \\
\quad \text { FR7300827 }\end{array}$ & 6,5 km en aval du barrage de Riète & $\begin{array}{l}600 \text { m } \\
\quad(160 \text { m de dénivelé) }\end{array}$ & $\begin{array}{l}\text { Tronçon court-circuité (barrage de Riète). } \\
\text { Hétérogénéité des faciès d'écoulement et des } \\
\text { substrats: } \\
\text { - courant rapide et vif, cascades et gros blocs } \\
\text { émergents; } \\
\text { - plats courants et profondes vasques au } \\
\text { substrat plus sableux et organique; } \\
\text { - radiers avec petits blocs, galets et graviers. }\end{array}$ \\
\hline $\begin{array}{l}\text { Oriège } \\
\quad \text { FR7300831 }\end{array}$ & Orlu & $\begin{array}{l}1350 \text { m } \\
\text { (170 m de dénivelé) }\end{array}$ & $\begin{array}{l}\text { Tronçon court-circuité (retenue d'En Beys). } \\
\text { Hétérogénéité des faciès d'écoulement: } \\
\text { - plats; } \\
\text { - radiers; } \\
\text { - forts dénivelés dans la hêtraie; } \\
\text { - zones élargies formant un réseau } \\
\quad \text { anastomosé avec des pertes d'eau. }\end{array}$ \\
\hline $\begin{array}{l}\text { Larrau } \\
\quad \text { FR7200790 }\end{array}$ & $\begin{array}{l}\text { Entre les lieux-dits Oste } \\
\text { et Barhenborda }\end{array}$ & $\begin{array}{l}350 \mathrm{~m} \\
(40 \mathrm{~m} \text { de dénivelé) }\end{array}$ & $\begin{array}{l}\text { En amont (une section) et en aval d'un barrage. } \\
\text { Hétérogénéité des faciès d'écoulement: } \\
\text { - radier sur substrat rocheux composé de } \\
\text { galets et de cailloutis; } \\
\text { - débits plus lents et profondeurs plus } \\
\text { importantes avec sédiments plus fins } \\
\text { d'aspects limoneux; } \\
\text { - débits beaucoup plus rapides de type «vif» } \\
\text { et «rapides» associés à des profondeurs } \\
\text { d'eaux variables. }\end{array}$ \\
\hline
\end{tabular}

\section{MATÉRIELS ET MÉTHODES}

\section{SiTES D'ÉTUDES}

Les sites ont été choisis de manière à proposer différents contextes et caractéristiques (Tableau 1):

- sites non perturbés par l'hydroélectricité: Salat (09),

Rébenty (11);

- site en partie perturbé par l'hydroélectricité: Nohèdes (66);

- sites perturbés par l'hydroélectricité: Aston (09), Oriège (09);

- site perturbé par l'hydroélectricité et en limite occidentale d'aire de répartition: Larrau (64).

\section{PROSPECTIONS ET COLLECTES DES FÈCES}

Les prospections pour la collecte des indices de présence, les fèces, ont été effectuées entre les mois d'août 2014 et novembre 2016, à hauteur de sept passages par site, répartis sur les périodes les plus propices pour la collecte de feces. Chaque site représentait un tronçon de $3 \mathrm{~km}$ sur lequel les prospections étaient réalisées sur six sections nommées "GAL" de 250 mètres, espacées les unes des autres par des sections non prospectées de 250 mètres. Les prospections se sont donc effectuées sur un linéaire cumulé d'1,5 km, en prospectant systématiquement de l'aval vers l'amont, de GAL1 vers GAL6. Toutes les feces jugées comme appartenant au Desman étaient collectées par les opérateurs sur chaque section prospectée. Les échantillons étaient prélevés à l'aide de gants pour éviter toute contamination, conservés séparément dans des micro-tubes contenant de l'éthanol à 95, puis stockés au congélateur avant envoi pour analyses au laboratoire de génétique. Chaque échantillon prélevé était géolocalisé avec une précision de 10 à 50 mètres afin de rendre les analyses de déplacements et de densités plus précises.

Tous les prospecteurs ont été formés aux prospections de feces de Desman. Seules deux personnes par site effectuaient les prospections sur toute la durée du suivi afin de supprimer le biais observateur d'une année à l'autre.

\section{ANALYSES GÉNÉTIQUES}

L'ADN des échantillons a été extrait à l'aide du QIAamp fast DNA stool Mini Kit de Qiagen. L'ensemble des échantillons a été analysé sur la base de deux approches complémentaires : 
TABLEAU 2. - Nombre total de fèces prélevées sur chaque site et chaque section «GAL».

\begin{tabular}{|c|c|c|c|c|c|c|c|}
\hline Cours d'eau & GAL_1 & GAL_2 & GAL_3 & GAL_4 & GAL_5 & GAL_6 & Total \\
\hline Salat & 21 & 22 & 10 & 24 & 11 & 5 & 93 \\
\hline Nohèdes & 38 & 58 & 34 & 18 & 32 & 18 & 198 \\
\hline Aston & 16 & 15 & 17 & 21 & 28 & 41 & 138 \\
\hline Oriège & 78 & 117 & 48 & 66 & 1 & 43 & 353 \\
\hline Larrau & 37 & 39 & 49 & 87 & 45 & 104 & 361 \\
\hline
\end{tabular}

- l'amplification et le séquençage d'un fragment du gène cytochrome oxydase 1 (CO1) de l'ADN mitochondrial afin d'identifier l'espèce ayant produit la crotte;

- le génotypage à partir de douze marqueurs microsatellites spécifiques pour Galemys pyrenaicus, afin d'effectuer les identifications individuelles.

Ces analyses ont été développées dans le laboratoire de génétique de la conservation de l'Université de Liège (Gillet et al. 2016).

Les douze marqueurs microsatellites ont été amplifiés par PCR en deux "mix multiplex». Une amorce de chaque locus microsatellite était marquée par un fluorochrome particulier. S'agissant d'échantillons prélevés de façon noninvasive, l'ADN est généralement de moindre qualité. Une approche multitube a donc été appliquée et les PCR ont été répétées au minimum trois fois pour chaque échantillon. Les produits PCR ont été analysés sur un séquenceur multicapillaire. Les génotypes multiloci ont été déterminés via le logiciel Genemapper 4.0 (Applied Biosystems). Les échantillons présentant au minimum sept loci amplifiés ont été retenus.

L'identification des génotypes individuels, pour rechercher un même individu sur des fecces différentes, été réalisée à l'aide du logiciel GIMLET (Valière 2002).

\section{RÉSULTATS}

Le site d'étude du Rébenty, dans l'Aude, a subi une pollution résultant en une dégradation importante de la biodiversité et en la disparition locale du Desman des Pyrénées pendant les années de suivi. Les sites considérés dans la suite de l'article ne seront donc plus qu'au nombre de cinq: Salat (non perturbé par l'hydroélectricité), Nohèdes (en partie perturbé par l'hydroélectricité), Aston et Oriège (perturbés par l'hydroélectricité) et enfin Larrau (perturbé par l'hydroélectricité et en limite occidentale d'aire de répartition).

\section{IDENTIFICATION DE L'ESPÈCE ET INDIVIDUALISATION}

Les résultats d'identification de l'espèce ont donné deux types de signaux:

- signal positif pour le Desman;

- aucune amplification, ni du gène $\mathrm{CO} 1$, ni des marqueurs microsatellites.

Les analyses génétiques peuvent également mener à l'identification d'une autre espèce mais dans le cadre de ce suivi, aucun échantillon envoyé à l'analyse génétique n'a abouti à ce résultat. Il est donc important de noter que chaque échantillon analysé génétiquement et n'aboutissant pas à l'identification du Desman des Pyrénées reflète un échantillon dont l'ADN est trop dégradé (aucune amplification) et non une erreur du prospecteur concernant l'espèce. L'analyse génétique permettant l'individualisation de l'échantillon n'est elle aussi possible que lorsque la qualité de l'ADN le permet.

\section{PRÉSENCE DE CROTTES PAR SECTION ET PAR SITE}

Le nombre de fèces prélevées est variable d'un site à l'autre et d'une section à l'autre (Tableau 2). L'Oriège est le cours d'eau où le plus de fecces ont été prélevées mais dispose d'une section (GAL_5) pour laquelle le nombre de fèces est quasinul. À l'inverse, le Salat est le cours d'eau disposant du plus faible nombre d'indices prélevés mais pour lequel ce nombre est le moins variable d'une section à l'autre.

Sur Nohèdes, les sections GAL_1 et GAL_2 sont celles qui se situent en aval de la centrale hydroélectrique mais leurs nombres d'indices de présence sont similaires à ceux des autres sections.

Sur le Larrau, la section GAL_6 se situe à l'amont du barrage et est celle possédant le plus grand nombre de fèces collectées.

\section{DÉPLACEMENT DES INDIVIDUS}

Au total, 43 individus différents ont pu être détectés grâce aux analyses génétiques (détails précisés en Annexe 1). Six d'entre eux ont été contactés deux à trois fois, permettant ainsi de comparer la localisation de leurs fèces grâce à leur géolocalisation et d'en déduire le déplacement minimal effectué par l'individu.

Sur le Salat, site non perturbé par l'hydroélectricité, deux individus ont été contactés à deux ou trois reprises. Pour l'un, deux fèces ont été collectées le même jour à seulement 10 mètres d'écart. Pour l'autre, deux de ses crottes ont également été collectées le même jour à 10 mètres d'écart, mais la troisième crotte lui appartenant a été prélevée plus de neuf mois après sur une section 710 mètres en aval.

Sur l'Aston, cours d'eau perturbé par l'hydroélectricité, un individu a été détecté à trois reprises lors d'un même passage. Ses fèces étaient espacées au maximum de 100 mètres.

Sur l'Oriège, cours d'eau également perturbé par l'hydroélectricité, un individu a été contacté deux fois à moins de deux mois d'écart et ses fèces ont été trouvées à 2,4 km de distance l'une de l'autre.

Sur le Larrau, en limite occidentale d'aire de répartition du Desman et sur un cours d'eau perturbé par l'hydroélectricité, 


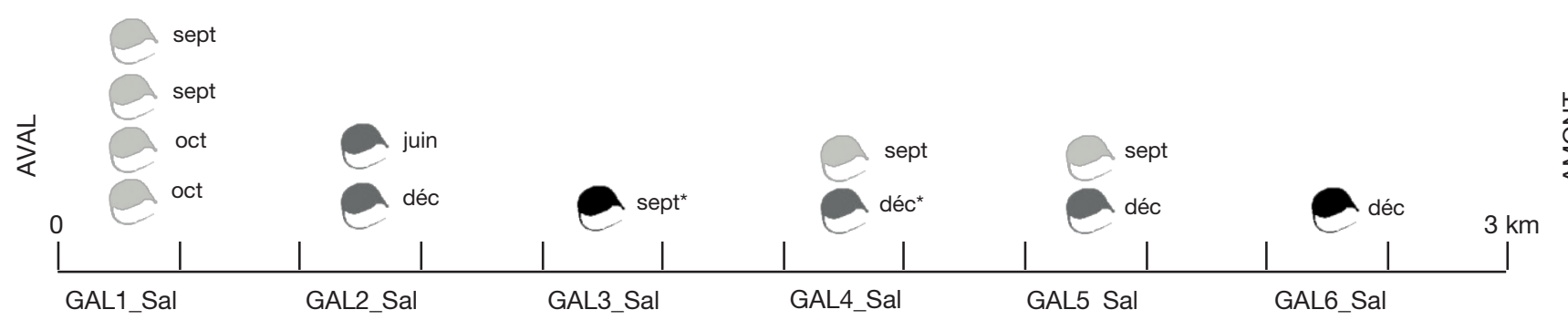

FIG. 1. - Répartition des individus génétiquement identifiés sur les sections «GAL» du tronçon de 3 km du Salat. Les couleurs représentent les années: en gris clair, 2014; en gris foncé, 2015 et en noir, 2016. Les mois de collecte de la crotte sont indiqués à côté du Desman concerné. *, même individu contacté à deux reprises mais sur deux sections différentes donc représenté deux fois.

deux individus ont été contactés deux fois. Dans les deux cas, leurs fèces respectives ont été trouvées le même jour à 20 et 70 mètres de distance.

NOMBRE D'INDIVIDUS PAR SECTIONS GAL ET PAR TRONÇON Grâce à la géolocalisation des crottes et à la date de récolte des échantillons, il est possible de positionner les individus sur le tronçon prospecté et de connaître le nombre de Desmans identifiés à un instant donné et sur une portion donnée.

Ainsi sur le Salat, onze individus distincts ont été identifiés. Une représentation graphique (Fig. 1) des Desmans contactés permet de visualiser les individus sur les différentes sections "GAL» du tronçon prospecté.

Le Salat possède ainsi quatre Desmans différents sur une seule portion de 250 mètres (GAL1_Sal), dont les fèces ont été prélevées entre septembre et octobre 2014, ou encore deux Desmans différents dont les fèces ont été prélevées sur une autre portion de 250 mètres (GAL2_Sal) entre juin et décembre 2015. En considérant tous les individus différents, onze Desmans distincts ont été contactés sur ce site donc onze individus sont présents sur $1,5 \mathrm{~km}$ de cours d'eau prospecté entre septembre 2014 et décembre 2016.

Les représentations graphiques des tronçons étudiés sont présentées en Annexe 2, sur le même modèle que la Figure 1 ci-dessus. En reprenant le modèle expliqué pour le Salat, il est possible de déterminer le nombre d'individus minimum se trouvant sur des portions de 250 mètres ou sur le tronçon prospecté, à savoir 1,5 km en cumulé (Tableau 3).

\section{DENSITÉ MINIMALE PAR PASSAGE}

\section{ET DENSITÉ MOYENNE MINIMALE PAR ANNÉE}

Grâce à l'individualisation des fèces, il est également possible de calculer une densité minimale pour chaque passage (nombre minimal d'individus $/ \mathrm{km}$ ) ainsi qu'une densité moyenne minimale par année.

Sur le Salat par exemple, quatre individus sont contactés en septembre 2014 sur 1,5 km de cours d'eau prospecté, soit 2,67 individus $/ \mathrm{km}$. En octobre 2014, deux individus sont contactés soit 1,33 individus/km (Fig. 1). Sur l'année 2014, la densité moyenne minimale est donc de 2.

Le Tableau 4 présente les résultats pour chaque passage, chaque année et chaque site. Ainsi, les densités minimales obtenues varient de 0,67 à 2,67 individus $/ \mathrm{km}$, et il en est de même pour les densités moyennes minimales obtenues par année.

\section{DISCUSSION}

Le nombre de fèces prélevées est variable d'un site à l'autre et au sein des sections d'un même site. Le Salat est le site pour lequel ce nombre est le plus faible mais également celui pour lequel la variabilité inter-sections est la plus faible. Hors il s'agit d'un site non perturbé par l'hydroélectricité. L'Oriège, à l'inverse, site perturbé par l'hydroélectricité, est celui ayant la plus grande variabilité du nombre de fèces entre les différentes sections mais celui possédant le plus grand nombre de fèces collectées. Enfin, sur le Larrau, il est intéressant de noter que la section GAL_6, seule section à l'amont du barrage, est celle pour laquelle le nombre de fèces collectées est le plus grand (104 contre 37 à 87 fèces prélevées sur les autres sections). Cependant, il est nécessaire de rappeler qu'aucun relevé d'habitat n'a été fait ici et qu'il est possible que ces disparités s'expliquent par la configuration des cours d'eau et le nombre de supports présents sur chaque site ou chaque section.

Les résultats des analyses génétiques effectuées sur les fèces de Desmans montrent une espèce non territoriale et nomade, comme évoqué dans différentes études (Némoz \& Bertrand 2009; Aymerich \& Gosálbez 2014; Gillet 2015; Escoda et al. 2018). En effet, plusieurs individus sont trouvés sur des portions restreintes de 250 mètres de cours d'eau.

Aussi, grâce aux analyses permettant l'individualisation des fèces, il est possible de donner des densités minimales de 0,67 à 2,67 individus $/ \mathrm{km}$. Ces densités sont variables entre les sites, quel que soit le contexte (hydroélectricité et répartition géographique) et au sein d'un même site entre les différents passages. Les densités moyennes minimales obtenues par année varient elles aussi selon les mêmes valeurs. Ces chiffres sont cohérents à ceux cités dans la bibliographie. En effet, Aymerich \& Gosálbez (2014) évoquent une densité moyenne de deux individus $/ \mathrm{km}$, valeur pouvant varier ponctuellement de 0 à 5-6 individus $/ \mathrm{km}$, grâce à leurs études menées de 2000 à 2009. Nores estime des densités allant de 2,8 \pm 0,2 à 7,3 $\pm 5,5$ individus/km (Nores 1992; Nores et al. 1998) sur quatre sessions de capture de quatre nuits, chacune durant l'été 1991. Gisbert \& García-Perea (2004), quant à eux, estiment cette densité à 3,2 à 5,5 individus/ $\mathrm{km}$. Enfin, une étude plus récente et similaire à la nôtre de Gillet et al. (2016) fait état de 28 Desmans identifiés sur $3 \mathrm{~km}$ de cours d'eau pendant 32 mois d'études mais ne cal- 
TABLEAU 3. - Répartition des individus distincts identifiés par analyse génétique et particularités sur les sections «GAL».

\begin{tabular}{lll}
\hline Cours d'eau & Nb d'individus distincts & Particularités \\
\hline Salat & 11 & $\begin{array}{l}\text { Quatre desmans sur GAL1 en 2014 } \\
\text { Deux desmans sur GAL2 en 2015 } \\
\text { Deux desmans sur GAL2 en 2014 } \\
\text { Deux desmans sur GAL3 en 2014 }\end{array}$ \\
Nohèdes & 5 & $\begin{array}{l}\text { Sept desmans en 2015 sur } 9 \text { présents entre 2014 et 2016 } \\
\text { Deux desmans sur GAL3 en 2015 } \\
\text { Deux desmans sur GAL4 en 2015 }\end{array}$ \\
Oriège & 9 & $\begin{array}{l}\text { Trois desmans sur GAL6 en } 2014 \\
\text { Larrau }\end{array}$ \\
\hline
\end{tabular}

TABlEAU 4. - Nombre minimal d'individus et densité minimale ( $\mathrm{Nb}$ individus/km) par passage ou sur l'année.

\begin{tabular}{|c|c|c|c|c|}
\hline Cours d'eau & Passage & $\begin{array}{c}\text { Nombre minimal } \\
\text { d'individus }\end{array}$ & Densité minimale & $\begin{array}{l}\text { Densité moyenne } \\
\text { minimale par année }\end{array}$ \\
\hline Salat & $\begin{array}{l}\text { Septembre } 2014 \\
\text { Octobre } 2014 \\
\text { Juin } 2015 \\
\text { Décembre } 2015 \\
\text { Septembre } 2016 \\
\text { Décembre } 2016\end{array}$ & $\begin{array}{l}4 \\
2 \\
1 \\
3 \\
1 \\
1\end{array}$ & $\begin{array}{l}2,67 \\
1,33 \\
0,67 \\
2 \\
0,67 \\
0,67\end{array}$ & $\begin{array}{l}2 \\
\overline{1} \\
\overline{1}, 33 \\
\overline{0}, 67 \\
-\end{array}$ \\
\hline Nohèdes & $\begin{array}{l}\text { Octobre2014 } \\
\text { Juillet } 2016\end{array}$ & $\begin{array}{l}4 \\
1\end{array}$ & $\begin{array}{l}2,67 \\
0,67\end{array}$ & $\begin{array}{l}2,67 \\
0,67\end{array}$ \\
\hline Aston & $\begin{array}{l}\text { Septembre } 2014 \\
\text { Juillet } 2015 \\
\text { Septembre } 2015 \\
\text { Novembre } 2015 \\
\text { Août } 2016\end{array}$ & $\begin{array}{l}1 \\
1 \\
3 \\
3 \\
1\end{array}$ & $\begin{array}{l}0,67 \\
0,67 \\
2 \\
2 \\
0,67\end{array}$ & $\begin{array}{l}0,67 \\
1,56 \\
- \\
- \\
0,67\end{array}$ \\
\hline Oriège & $\begin{array}{l}\text { Début octobre } 2014 \\
\text { Fin octobre } 2014 \\
\text { Début octobre } 2015 \\
\text { Fin octobre } 2015 \\
\text { Août } 2016 \\
\text { Octobre } 2016\end{array}$ & $\begin{array}{l}3 \\
2 \\
1 \\
1 \\
2 \\
1\end{array}$ & $\begin{array}{l}2 \\
1,33 \\
0,67 \\
0,67 \\
1,33 \\
0,67\end{array}$ & $\begin{array}{l}1,67 \\
- \\
0,67 \\
- \\
1 \\
-\end{array}$ \\
\hline Larrau & $\begin{array}{l}\text { Septembre } 2014 \\
\text { Octobre } 2014 \\
\text { Août } 2015 \\
\text { Novembre } 2015 \\
\text { Juillet } 2016\end{array}$ & $\begin{array}{l}2 \\
2 \\
1 \\
3 \\
1\end{array}$ & $\begin{array}{l}1,33 \\
1,33 \\
0,67 \\
2 \\
0,67\end{array}$ & $\begin{array}{l}1,33 \\
- \\
1,33 \\
\overline{0,67}\end{array}$ \\
\hline
\end{tabular}

cule pas de densités. Les captures réalisées lors de cette étude permettent quant à elles d'identifier 11 individus sur trois sections de 300 mètres (soit 900 mètres au total). Ce travail montre également la capacité de déplacement des Desmans, notamment sur l'Oriège où un même individu a parcouru plus de $2 \mathrm{~km}$ vers l'aval en moins de deux mois, étayant ainsi les résultats des études menées sur l'Aude (Gillet et al. 2016) avec des déplacements de Desmans de près de $18 \mathrm{~km}$ en un an ou encore sur le Vicdessos avec des déplacements de $6,2 \mathrm{~km}$ en moins de trois jours.

Concernant la méthodologie utilisée ici, malgré la variabilité du nombre de fèces collectées d'une année à l'autre sur un même site, elle reste un protocole facilement applicable et transposable sur l'ensemble de l'aire de répartition du Desman des Pyrénées, malgré les aléas de qualité d'ADN des échantillons rendant les analyses génétiques parfois vaines. En effet, son caractère non invasif en fait une méthode privilégiée par rapport aux captures, plus coûteuses en moyens humains et moins efficaces pour inventorier l'espèce (Poncet et al. 2021). De plus, les résultats des analyses génétiques ont montré la rigueur des prospections car aucune crotte analysée supposée comme appartenant au Desman par le prospecteur n'a été identifiée comme appartenant à une autre espèce. Il serait cependant intéressant d'appliquer ces analyses génétiques sur des linéaires plus importants et sur des périodes de temps plus longues avec des prospections régulières afin de comprendre la dynamique des populations. La plus grande régularité des prospections pourrait également permettre de diminuer les pertes de données lors des analyses génétiques dues à la dégradation de l'ADN de l'échantillon. Enfin, une analyse génétique permettant l'établissement d'un lien de parenté plus poussé (parent-enfant, fratrie, parenté plus éloignée), comme étudiée par les chercheurs espagnols (Escoda et al. 2018) aurait peut-être expliqué les dispersions ou les regroupements de Desmans sur certaines portions de cours d'eau. 


\section{CONCLUSION}

Les nombres de feces prélevées diffèrent d'un site à l'autre, mais également d'une section à l'autre au sein d'un même site, sans répartition particulière de ces derniers en fonction du contexte considéré, hormis qu'il soit plus faible mais plus constant entre les différentes sections pour le Salat, seul site non perturbé par l'hydroélectricité (hors Rébenty), à l'inverse de l'Oriège, tronçon court-circuité.

Concernant le protocole utilisé, cette étude a montré l'efficacité des prospections avec $100 \%$ des échantillons prélevés et considérés comme appartenant au Desman des Pyrénées identifiés comme tels après analyses génétiques pour les échantillons possédant une qualité d'ADN suffisante pour parvenir à l'espèce. Ainsi, dans le cadre d'inventaires ayant pour but la détection de l'espèce seulement, les prospections sont suffisantes si elles sont réalisées par les personnes compétentes et en respectant le protocole établi dans le cadre du programme LIFE+ Desman (Charbonnel et al. 2017).

Les analyses génétiques ont quant à elles confirmé le caractère nomade et non territorial du Desman des Pyrénées, avec notamment des déplacements de plus de $2 \mathrm{~km}$ en moins de deux mois sur l'Oriège (perturbé par l'hydroélectricité), ou encore des densités minimales de quatre individus sur une portion de 250 mètres seulement et sur deux mois sur le Salat (non perturbé).

Les densités de populations estimées par passage ou par année ne montrent quant à elles pas de réelle différences entre les sites perturbés ou non, ni par rapport à la position géographique du cours d'eau (limite occidentale d'aire de répartition).

Enfin, cette étude a également confirmé l'impact d'un incident de pollution sur les populations de Desman, comme pour le Rébenty, site autrefois connu pour héberger le Desman mais très fortement perturbé, au point d'amener l'espèce à disparaître durant la durée de l'étude.

La capacité de déplacement des Desmans et la difficulté de contact de l'espèce (variabilité du nombre de fèces d'une année sur l'autre) rendent sa prise en compte nécessaire sur des cours d'eau favorables, même s'il semble absent à un instant donné. En effet, certains cours d'eau hébergeant historiquement le Desman des Pyrénées mais sur lesquels l'espèce n’a pas été détectée récemment restent tout de même des habitats potentiels et favorables à l'accueil d'individus capables de déplacements importants. Cela pointe aussi l'importance de la continuité écologique et de son maintien dans les projets d'aménagements de cours d'eau, afin de ne pas fragmenter les populations en empêchant les flux d'individus et le mélange des populations génétiques.

\section{Remerciements}

Nous remercions tous les partenaires ayant participé aux prospections: I'ANA-CEN Ariège, la Fédération Aude Claire, la Fédération des Réserves naturelles Catalanes, le CEN Nouvelle-Aquitaine et l'Office français de la Biodiversité. Grâce à eux, nous avons pu collecter de précieux échantillons pour réaliser cette étude.
Nous remercions également les deux rapporteurs Patrick Haffner et Audrey Savouré-Soubelet pour leur relecture et leur contribution à l'amélioration de cet article.

\section{RÉFÉRENCES}

Aymerich P. \& GosÁlbez J. 2014. - El desmán ibérico Galemys pyrenaicus en los Pireneos meridionales. Munibe Monographs. Nature Series 3: 37-77. https://doi.org/10.21630/ mmns.2014.3.04

Aymerich P. \& Gosálbez J. 2015. — Evidencias de regresión local del desmán ibérico (Galemys pyrenaicus) en los Pirineos meridionales. Galemys 27: 31-40. https://doi.org/10.7325/ Galemys.2015.A4

AyMerich P. \& GosÁlbez J. 2018. — Regresión reciente y general del desmán ibérico en su límite noreste de distribución. Galemys 30: 1-10. https://doi.org/10.7325/Galemys.2018.A2

Charbonnel A. 2015. - Influence multi-échelle des facteurs environnementaux dans la répartition du Desman des Pyrénées (Galemys pyrenaicus) en France. Thèse de l'Université de Toulouse, $260 \mathrm{p}$.

Charbonnel A., Blanc F., Némoz M., Buisson L., LafFaille P., Bodo A., Fournier-Chambrillon C., Fournier P., Fropier N., Lacaze V., Le Roux B., Poncet E., PontcharRAUD L. \& TRIBOLET L. 2017. — Outils techniques pour la prise en compte du Desman des Pyrénées dans les procédures d'évaluations environnementales. Livret 2 - Outil cartographique d'alerte et cahier des charges pour la réalisation d'inventaires du Desman des Pyrénées. Conservatoire d'Espaces naturels de Midi-Pyrénées, Toulouse, $28 \mathrm{p}$.

Chora S. C. \& Quaresma C. M. 2001. - Toupeira-de-agua, Galemys pyrenaicus : padrôes de ocupação espacio-temporale capacidade de transposição da mini-hidrica de Fraguas (Rio Paiva, Bacia Do Douro). $2^{\circ}$ Congreso Nacional de Conservacion de la Naturaleza (ICN), Lisbonne.

Escoda L., FernándeZ-GonZÁlez A. \& Castresana J. 2018. Quantitative analysis of connectivity in populations of a semi-aquatic mammal using kinship categories and network assortativity. Molecular Ecology Resources 19: 310-326. https:// doi.org/10.1111/1755-0998.12967

Esnaola A., GonZÁleZ-Esteban J., Elosegi A., Arrizabalaga-EsCudero A. \& Aihartza J. 2018. — Need for speed: Preference for fast-flowing water by the endangered semi-aquatic Pyrenean desman (Galemys pyrenaicus) in two contrasting streams. Aquatic Conservation: Marine and Freshwater Ecosystems 28 (3): 1-10. https://doi.org/10.1002/aqc.2893

GILLET F. 2015. - Génétique et biologie de la conservation du Desman des Pyrénées (Galemys pyrenaicus) en France. Thèse de l'Université de Toulouse, $228 \mathrm{p}$.

Gillet F., Le Roux B., Blanc F., Bodo A., Fournier-Chambrillon C., Fournier P., Jacob F., Lacaze V., Némoz M., Aulagnier S. \& Michaux J. R. 2016. - Genetic monitoring of the endangered Pyrenean desman (Galemys pyrenaicus) in the Aude River, France. Belgian Journal of Zoology 146 (1): 44-52. https://doi.org/10.26496/bjz.2016.38

GisBert J. \& GarCÍA-PEREA R. 2004. - Estudio cuantitativo sobre la población de Desmán Ibérico en el valle del Ambroz y del Topillo de Cabrera en el norte de Extremadura. Junta de Extremadura, Mérida, $400 \mathrm{p}$.

Gisbert J. \& GARCÍA-PEREA R. 2014. - Historia de la regresión del desmán ibérico Galemys pyrenaicus (E. Geoffroy Saint-Hilaire, 1811) en el Sistema Central (Península Ibérica). Munibe Monographs. Nature Series 3: 19-35. https://doi.org/10.21630/mmns.2014.3.03

LiM M. 2015. — Étude de l'incidence des lâchers d'eau sur le comportement et la survie du Desman des Pyrénées (Galemys pyrenaicus), en période de reproduction par la méthode de suivi par radiopistage. 
Mémoire, INP-ENSAT, Toulouse, 83 p.

Melero Y., Aymerich P., Luque-Larena J. J. \& Gosàlbez J. 2011. - New insights into social and space use behaviour of the endangered Pyrenean desman (Galemys pyrenaicus). European Journal of Wildlife Research 58: 185-193. https://doi.org/10.1007/ s10344-011-0561-7

Melero Y., Aymerich P., Santulli G. \& Gosàlbez J. 2014. Activity and space patterns of Pyrenean desman (Galemys pyrenaicus) suggest non-aggressive and non-territorial behavior. European Journal of Wildlife Research 60: 707-715. https://doi. org/10.1007/s10344-014-0838-8

Némoz M. \& Bertrand A. 2009. - Plan national d'Actions en faveur du Desman des Pyrénées (Galemys pyrenaicus) 2009-2015. Ministère de l'Écologie, de l'Énergie, du Développement durable et de la Mer, Paris, $160 \mathrm{p}$.

Nores C. 1992. - Estudio previo del area de distribucion del desman en la Penincula Iberica. Departamento de biologia de organismos et sistema, Universitad de Oviedo, $18 \mathrm{p}$.

Nores C., Ojeda F., Ruano A., Villate I., Gonzalez J., CANO J. M. \& GARCIA E. 1998. — Estimating the population density of Galemys pyrenaicus in four Spanish rivers. Journal of Zoo$\operatorname{logy} 246$ (4): 454-457. https://doi.org/10.1111/j.1469-7998.1998. tb00179.x

Poncet E., Némoz M., Blanc F. 2017. — Outils techniques pour la prise en compte du Desman des Pyrénées dans les procédures d'évaluations environnementales. Livret 1 - État des connaissances sur le Desman des Pyrénées. Conservatoire d'Espaces naturels de Midi-Pyrénées, Toulouse, $20 \mathrm{p}$.

Poncet E., Blanc F., Lim M., Marc D. \& Némoz M. 2021. Desman des Pyrénées (Galemys pyrenaicus É. Geoffroy SaintHilaire, 1811) 2011-2018: bilan et retours d'expérience sur huit années de capture dans les Pyrénées françaises. Naturae 2021 (3): 35-47. https://doi.org/10.5852/naturae2021a3

Quaglietta L., Paupério J., Martins F., Alves P. C. \& Beja P. 2018. - Recent range contractions in the globally threatened Pyrenean desman highlight the importance of stream headwater refugia. Animal Conservation 21 (6): 1367-9430. https://doi. org/10.1111/acv.12422

STONE R. D. 1985. - Home range movements of the Pyrenean desman (Galemys pyrenaicus) (Insectivore: Talpidae). Zeitschrift für Angewandte Zoologie 72 (1-2): 25-36.

UICN FRANCE, MNHN, SFEPM \& ONCFS. 2017. — La Liste rouge des espèces menacées en France - Chapitre Mammiferes de France métropolitaine. UICN France, Muséum national d'Histoire naturelle, Paris, $16 \mathrm{p}$.

VALIÈRE N. 2002. - GIMLET: a computer program for analysing genetic individual identification data. Molecular Ecology Notes 2 (3): 1471-1478. https://doi.org/10.1046/j.1471-8286.2002.00228.x-i2

Soumis le 9 mai 2020; accepté le 17 janvier 2021; publié le 4 aồt 2021. 


\section{ANNEXES}

ANNEXE 1. - Identification des individus distincts.

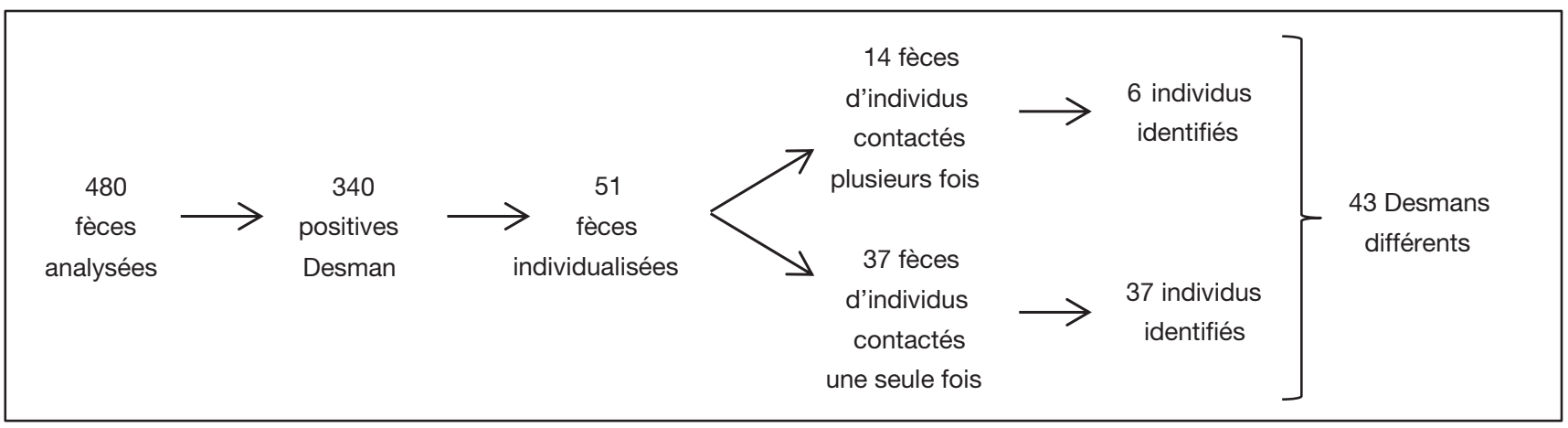

ANNEXE 2. - Répartition des individus sur les les sections «GAL» des différents tronçons étudiés: A, Salat; B, Nohèdes; C, Aston; D, Oriège; E, Larrau. Les couleurs représentent les années: en gris clair, 2014; en gris foncé, 2015 et en noir, 2016. Les mois de collecte des crottes sont indiqués à côté du Desman concerné. *, même individu contacté à deux reprises mais sur deux sections différentes ou sur deux années différentes donc représenté deux fois.
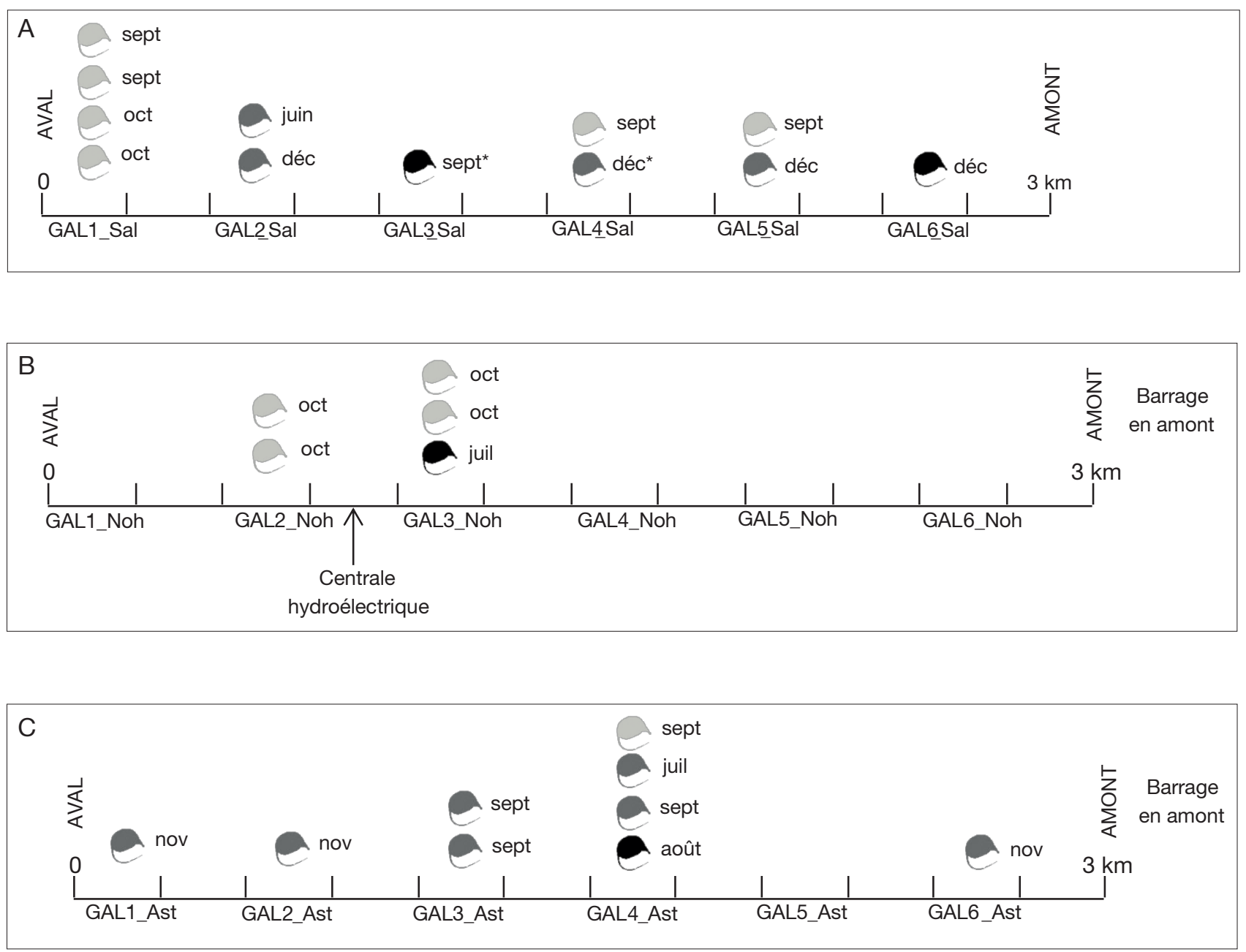
ANNEXE 2. - Suite.

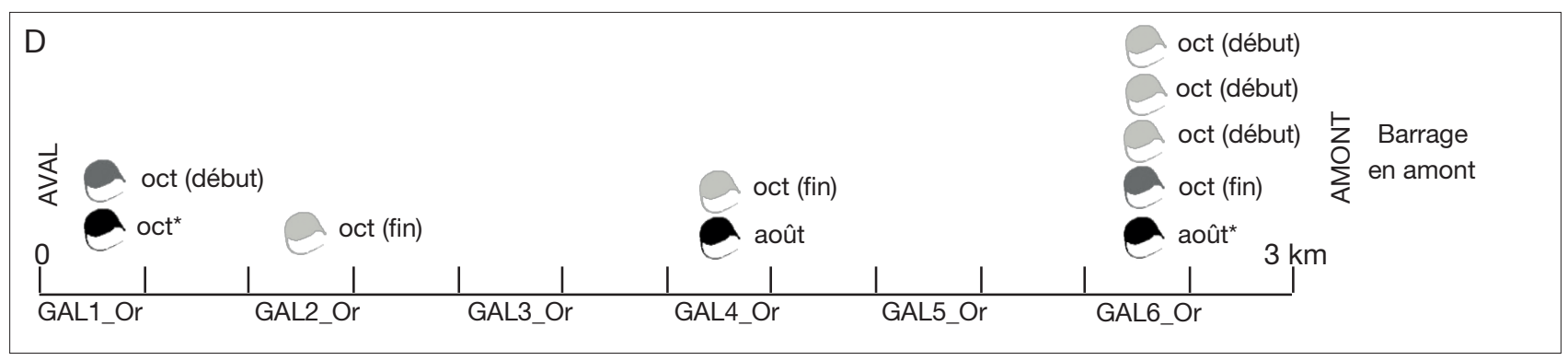

(a) sept

